\title{
To study the determinants of falls, fractures and frailty in elderly attending HSK hospital and research centre Bagalkot - an observational study
}

\author{
Sachin V Desai ${ }^{1}$, Santhoshkumar R Naik ${ }^{2, *}$, Ashok S Dorle ${ }^{3}$ \\ ${ }^{1}$ Assistant Professor, ${ }^{2}$ Post Graduate, ${ }^{3}$ Professor and Head, Dept. of Community Medicine, S.N.M.C and HSK and RC Bagalkot, \\ Karnataka, India \\ *Corresponding Author: \\ Email: santhoshkumarrnaik@gmail.com
}

\begin{abstract}
Introduction: Population ageing is a global phenomenon. Ageing is a physiological process that starts from birth, continues throughout life and ends with death. In India, according the census 2011, there are nearly 104 million elderly persons. 53 million are females and 51 million are males. Assessment of falls and the determining factors leading to falls gives us the corrective measures to be taken to avoid falls among elderly.

Objective: To study the determinants of falls, fractures and frailty among elderly visiting OPD/IPD in a tertiary care HSK hospital in Bagalkot.

Materials and Methods: An observational study conducted in HSK hospital, among 60 Elderly on falls, fractures and frailty visiting IPD/OPD. Information on socio-demographic data, history of falls, fractures and the determinants of falls, fractures and frailty details were collected with a pre-designed, pre-tested, structured questionnaire. Chi square test was applied to look for the association between the determinants and age and gender.

Results: Among 60 elderly populations $50(83.33 \%)$ were between 60-74 years age and $10(16.66 \%)$ were above 75 years age. Among 50, $38(63.33 \%)$ were females and 22(36.66\%) males. Hypertension, vertigo, giddiness, use of walker, showed statistically significant association between age and the determinants of falls. Also, other determinants like, falls during transferring; falls due to imbalance, slips also had association.

Conclusion: Falls are very common among the elderly. The findings provide us with an opportunity to understand the risk factors still better, and need for rehabilitation, implement policies to reduce the risk of falls leading to fractures and frailty.
\end{abstract}

Keywords: Elderly, Determinants, Falls \& Fractures, Frailty.

\section{Introduction}

"Population aging is a triumph of humanity but also a challenge to society". Globally, the number of persons over 60 years is growing at a faster pace than any other age group. The number was estimated to be 688 million in 2006, now projected to grow to almost a billion by $2050 .{ }^{1}$ In India, according to population census 2011, there are nearly 104 million elderly persons. 53 million females and 51 million are males. There is rise in elder population in India too as from $5.6 \%$ in 1961 to $8.6 \%$ in current scenario. Generally, aging, though; reflects the longer life, partly healthier lives of the elder persons, at the same time it is associated with chronic and degenerative multisystem and functional disorders leading to disability and limiting the quality of life. Along with this, as people age, there are chances to become frailer, thereby increased chances of deterioration with decreased recovery from this frailty. Although, there is agreement that frailty is a much useful concept for clinical researchers, no complete definition available. But accordingly, it has been defined as per the need and different ones are available in the literature depending on the features used to describe it. So, various operational definitions are available to explain the syndromic nature of the condition making the things more complex. The prevalence of frailty among elderly ranges from $33 \%$ to $88 \%$ depending on the criteria used.
Elderly can be classified according to the grouping done by ageing as starting from young old (6074years), old (75-89 years), very old (90-99 years), and centenarians $\left(>100\right.$ years). ${ }^{2}$ With the rapidly increasing population aging in western societies, frailty is set to reach the epidemic levels in coming few decades. In India, though frailty is much higher than expected, no such studies and data are available.

Frailty is defined as the loss of a person's ability to withstand minor environmental stresses because of reduced reserves in the physiological function of several organ systems. In functional terms, it is defined as dependence on others for activities of daily living (ADLs), ${ }^{3,4}$ bathing, dressing, feeding, continence, toileting, and mobility. The term 'frail' is contemplated to identify vulnerable elderly people at high risk of adverse outcomes including falls, morbid disability, hospitalization and mortality. The most widely accepted criteria are those of Fried et al, who define Frailty Syndrome ${ }^{5}$ as including three or more of the following: weakness, slow walking, speed, self-reported exhaustion, low physical activity, and unintentional weight loss.

Aging in the people leads to decreased strength, vision, proprioception, leading to reduced balance and altered gait leading to falls. Falls are commonly defined as "inadvertently coming to rest on the ground, floor or other lower level, excluding intentional change in position to rest in furniture, wall or other objects. 
The five giants of geriatric $^{6}$ identified as immobility, instability (falls), incontinence, intellectual impairment and iatrogenic. Among these, falls amount to be a major problem in the elderly; causing injuries, dependence, psychological difficulties, and social isolation. According to the centres for disease control and prevention (CDC), falls are the leading cause of injury among adults over the age of 65 , and each year one in three older adults, report having had a fall.

$20 \%-30 \%$ of injuries among the elderly are due to falls, and also contributes to $10 \%-15 \%$ of all emergency department visits. Falls is also responsible for $50 \%$ of injury-related hospitalization among people of 65 years and above ${ }^{7}$

Elderly are more susceptible to falls and related injury because of a high prevalence of clinical diseases (e.g. osteoporosis) and age-related physiological changes (e.g. slowed protective reflexes) that make even a relatively mild fall particularly dangerous. Also, recovery from fall injury is often delayed in older persons, which in turn increases risk of subsequent falls through reconditioning.

Falls in elderly can occur due to many reasons. In internal and external environment can pose as a risk factor for falls in elderly. Many other studies have shown that falls are dependent on many epidemiological and socio-demographic factors. There are lack of studies and research to identify the areas of key concern and to plan policies to address the issues. In most of the cases, falls are preventable. Prevention of falls is of utmost importance because they lead to considerable mortality, morbidity and suffering for older people and their families, and incur social costs due to hospital and nursing home admissions. Thus, identifying the risk factors and initiating plans of care to address these factors, falls can be prevented which will help in understanding their causal relationship and hence plan the preventive measures. This study was conducted in a tertiary care hospital with an objective to assess the determinants of falls, fractures and frailty in elderly visiting OPD/IPD in a tertiary care HSK hospital in Bagalkot.

\section{Materials and Methods}

The present observational study was carried out in out-patient and in-patient wards of Orthopaedics, Medicine, Surgery, Psychiatry, ENT, Ophthalmology departments of SNMC HSK Hospital Bagalkot. The study was done for a period of two months from July 2017 to August 2017. Elderly visiting OPD/IPD in a tertiary care hospital were included in the study. Taking prevalence of falls in elderly as 32\% from reviewed literature by Tinetti ME et al., ${ }^{8}$ the required sample came out to be 60 . Systematic random sampling was adopted to select the cases for the study from the hospital. Elderly 60 years and above visiting the OPD/IPD of above mentioned departments with present and or past history of falls/fractures or is under a caregiver were considered eligible for the study. Likewise, elderly under the required age who did not give consent for the study as well as those having metabolic bone disorders or any other congenital deformities were not taken for the study.

Each of the respective OPDs/IPDs was visited by the interviewer and interviewed the selected study participants using the pretested structured questionnaire in their own vernacular language after obtaining the consent. Permission to conduct the study was taken from the Institutional Ethics Committee. Sociodemographic details were taken; socio-economic status was assessed using modified B.G. Prasad Classification. Information about self-reported or physician diagnosed chronic health conditions, medication use, and current use of alcohol or tobacco was recorded. To know or confirm the use of medication by participants, they were asked to show the medicines or blister packs. Medical records, if available also seen.

History of presence or absence of risk factors for falls, fractures assessed. History of falls in the past 12 months was asked. Details regarding fall such as time and place of fall, severity, resulting the injury, part of the body injured, disability, hospitalization and treatment were recorded.

General physical examination and vitals addressed. Assessment of vision, hearing, functional disability and balance was done. For assessing vision, Snellen chart was used. For hearing assessment, the WHO grades of hearing impairment ${ }^{9}$ were used and Grades 2,3 , and 4 were considered as hearing impairment. For assessment of gait, get up and go test was used while body balance was assessed by Romberg's test. ${ }^{9}$

\section{Components assessed in studying the determinants} of falls were -

1. Health status at the time of fall - It was assessed as either presence or absence of these morbidities: Hypertension, Parkinson's disease, joint pain, memory loss/cognitive impairment, diabetes mellitus, vertigo/giddiness, $\mathrm{H} / \mathrm{O}$ fractures, depression, seizures, antihistamines

2. Mobility aids during falls, in older adults

Assessed as used or not - Walking stick, Walker, Staff personnel (in case of presence of care giver)

3. Activity during the time of fall

Assessed using these set of questions during which lead to fall - Bathing, housework, climbing stairs, transferring, ambulation

4. Location of fall -

Place of fall assessed - Bathroom, outside house, other rooms inside the house, street/road, threshold at the door entrance

5. Causes of falls -

Reasons for falls assessed structurally by interview - Hypertension, dizziness, imbalance, slips, trips, 
vehicular accidents, if no other cause could be specified

6. Injuries sustained due to fall -

Types of injuries assessed - Abrasion, contusion, fractures, dislocation of joints, sprains

7. Treatment received - First aid, hospital admission, OPD consultation

8. Fears of falls, thus restriction of normal daily activities- Assessed the level of activity.

\section{Operational Definitions}

Falls: An event that results in a person coming to rest inadvertently on the ground or floor or other lower level Substance abuse: Consumption of tobacco/alcohol was taken into consideration for at least 3 days in a week.

Knee pain/osteoarthritis: Current knee pain or physician-diagnosed osteoarthritis in the knee joint.

Disability: Inability or limitation in performing socially defined activity and roles expected of individuals within a social and physical environment. It included any impairment, activity limitation, and participatory restriction.

Statistical Analysis: All the collected data was entered in the MS Excel 2007, and statistical analysis was done using IBM SPSS software version 20 (trial version). Descriptive analysis was done, and the association was found between the dependent and independent variables using chi-square test and $\mathrm{p}<0.05$ was taken as significant.

\section{Results}

Among 60 geriatric participants, $50(83.33 \%)$ were young old (60-74years age) and $10(16.67 \%)$ were old elderly (>75 years age). Among 50 young old participants males were $36 \%$ and females $64 \%$. Similarly, $36 \%$ males and $40 \%$ females in old elderly participants most of them were illiterate $(63.33 \%)$, and $10 \%$ were educated up to high school. $55 \%$ were in Class IV socio economic class and $45 \%$ were in Class $\mathrm{V}$ socio economic Class. Most of them were in 3 generation family (70\%). Only 5\% were in nuclear family.

In studying the determinants of falls; when their association was assessed with age, $28 \%$ young old and $60 \%$ old elderly had fall with hypertension. $52 \%$ of the old elderly had joint stiffness that was the reason for their fall. $72 \%$ young old and $90 \%$ of the old elderly were having vertigo making it as a reason for their falls. Antihistamines as a factor for fall were $04 \%$ and $10 \%$ among young old and old elderly participants. Not using walker as a one of the mobility aids (cane/walking stick, walker, and staff/personnel) lead to fall among $76 \%$ of young old and $100 \%$ of old elderly and is statistically significant.

Geriatric study participants had fall in one or more activities like $66.67 \%$ during ambulation followed by involving in housework $(38.33 \%)$, then, bathing, transferring each 5\%, 3.33\% during climbing stairs.

$10 \%$ of the old elderly were having bath at the time of fall. Whereas, $10 \%$ of the young old had fall in bathroom $50 \%$ of the young old sustained fall inside the house in other rooms, followed by $42 \%$ outside the house. $20 \%$ on the street/road and $18 \%$ each sustained fall at threshold of the door entrance and vehicle related. Among the old elderly, $60 \%$ had fall in other rooms inside the house, followed by $40 \%$ outside the house, $20 \%$ each on the street/road and threshold at door entrance.

Among young old participants, imbalance was main cause for falls $(68 \% ; \mathrm{p}=0.009)$, followed by slips $(60 \%)$, dizziness $(34 \%)$. Hypertension as a morbidity leading to fall was seen among $18 \%$ young old participants and is statistically significant $(\mathrm{p}=0.005)$.

There were injuries sustained following falls among the study participants; among young old, 54\% sustained fractures, followed by sprains (46\%), and $18 \%$ dislocation of joints and $10 \%$ with contusions. Among old elderly, 50\%

Out of 60 study participants, previous history of falls was observed in $32(53.3 \%)$. When repeated history or a past history of fall was analyzed across various determinants, it was found that; vertigo, giddiness, dizziness, imbalance among the study participants had association with repeated history of fall. Whereas, though hypertension a common comorbidity lead to fall $(73.3 \%)$ did not show statistical significance. Antihypertensive drugs used by the study participants, might have caused imbalance or dizziness or vertigo which leads to fall along with age related degenerative changes in the brain of geriatric study participants.

Table1: Socio-demographic variables of study participants

\begin{tabular}{|l|c|c|c|}
\hline Variables & Male (\%) & Female (\%) & Total (\%) \\
\hline Age & $18(36)$ & $32(64)$ & $50(100)$ \\
\hline Young old (60-74 years) & $04(40)$ & $06(60)$ & $10(100)$ \\
\hline Old elderly (>75 years) & $11(28.9)$ & $27(71.1)$ & $38(100)$ \\
\hline Education & $05(31.2)$ & $11(68.8)$ & $16(100)$ \\
\hline Illiterate & $06(100)$ & $00(00)$ & $06(100)$ \\
\hline Primary School & \\
\hline High School &
\end{tabular}




\begin{tabular}{|l|c|c|c|}
\hline Farmer & $17(94.4)$ & $01(5.6)$ & $18(100)$ \\
\hline Mechanic & $01(100)$ & $00(00)$ & $01(100)$ \\
\hline Labourer & $02(100)$ & $00(00)$ & $02(100)$ \\
\hline Housewife & $00(00)$ & $36(100)$ & $36(100)$ \\
\hline Retired supervisor & $01(100)$ & $00(00)$ & $01(100)$ \\
\hline Weaver & $01(50)$ & $01(50)$ & $02(100)$ \\
\hline Socio-Economic Status & $13(39.4)$ & $20(60.6)$ & $33(100)$ \\
\hline Class IV & $09(33.3)$ & $18(66.7)$ & $27(100)$ \\
\hline Class V & $19(34.5)$ & $36(65.5)$ & $15(100)$ \\
\hline Type of family & $02(33.3)$ & $02(66.7)$ & $03(100)$ \\
\hline Joint & $01(42.2)$ & $00(00)$ & $42(100)$ \\
\hline Nuclear & $02(15.3)$ & $13(86.7)$ & $15(100)$ \\
\hline 3-Generation & $01(33.3)$ & $02(66.7)$ & $03(100)$ \\
\hline Type of house & $19(42.2)$ & $23(54.8)$ & $42(100)$ \\
\hline Kuccha & $17(50)$ & $17(50)$ & $34(100)$ \\
\hline Pucca & $05(19.2)$ & $21(80.8)$ & $26(100)$ \\
\hline Semi-pucca
\end{tabular}

Table 2: Age with determinants of falls

\begin{tabular}{|c|c|c|c|c|c|c|}
\hline \multirow{2}{*}{\multicolumn{2}{|c|}{$\begin{array}{l}\text { Age with Determinants of } \\
\text { falls }\end{array}$}} & \multicolumn{2}{|c|}{ Young old } & \multicolumn{2}{|c|}{ Old elderly } & \multirow[t]{2}{*}{$P$ value } \\
\hline & & $\mathbf{N}$ & $(\%)$ & $\mathbf{N}$ & $(\%)$ & \\
\hline \multicolumn{7}{|c|}{ Health Status at the time of fall } \\
\hline \multirow{2}{*}{$\begin{array}{l}\text { Hypertension } \\
\text { (giddiness) }\end{array}$} & Yes & 14 & 28 & 06 & 60 & \multirow[t]{2}{*}{0.057} \\
\hline & No & 36 & 72 & 04 & 40 & \\
\hline \multirow{2}{*}{$\begin{array}{l}\text { Parkinson's } \\
\text { disease }\end{array}$} & Yes & 01 & 02 & 00 & 00 & \multirow[t]{2}{*}{0.544} \\
\hline & No & 49 & 98 & 10 & 100 & \\
\hline \multirow{2}{*}{$\begin{array}{l}\text { Joint pain } \\
\text { (STIFFNESS) }\end{array}$} & Yes & 24 & 48 & 26 & 52 & \multirow[t]{2}{*}{0.908} \\
\hline & No & 05 & 50 & 05 & 50 & \\
\hline \multirow[t]{2}{*}{ Memory loss } & Yes & 05 & 10 & 01 & 10 & \multirow[t]{2}{*}{1.000} \\
\hline & No & 45 & 90 & 09 & 90 & \\
\hline \multirow{2}{*}{$\begin{array}{l}\text { Diabetes Mellitus } \\
\text { (peripheral } \\
\text { neuropathy) }\end{array}$} & Yes & 05 & 10 & 01 & 10 & \multirow[t]{2}{*}{1.0000} \\
\hline & No & 45 & 90 & 09 & 10 & \\
\hline \multirow{2}{*}{$\begin{array}{l}\text { Vertigo/ } \\
\text { Giddiness }\end{array}$} & Yes & 36 & 72 & 09 & 90 & \multirow[t]{2}{*}{0.194} \\
\hline & No & 14 & 28 & 01 & 10 & \\
\hline \multirow[t]{2}{*}{$\mathrm{H} / \mathrm{O}$ fractures } & Yes & 14 & 28 & 02 & 20 & \multirow[t]{2}{*}{0.593} \\
\hline & No & 36 & 72 & 08 & 80 & \\
\hline \multirow[t]{2}{*}{ Depression } & Yes & 01 & 02 & 00 & 00 & \multirow[t]{2}{*}{0.652} \\
\hline & No & 49 & 98 & 10 & 100 & \\
\hline \multirow[t]{2}{*}{ Seizures } & Yes & 00 & 00 & 01 & 10 & \multirow[t]{2}{*}{0.055} \\
\hline & No & 50 & 100 & 09 & 90 & \\
\hline \multirow[t]{2}{*}{ Antihistamines } & Yes & 02 & 04 & 01 & 10 & \multirow[t]{2}{*}{0.468} \\
\hline & No & 48 & 96 & 09 & 90 & \\
\hline \multicolumn{7}{|c|}{ Mobility aids during falls } \\
\hline \multirow{2}{*}{$\begin{array}{l}\text { Cane/ } \\
\text { Walking stick }\end{array}$} & Yes & 20 & 40 & 06 & 60 & \multirow[t]{2}{*}{0.246} \\
\hline & No & 30 & 60 & 04 & 40 & \\
\hline \multirow[t]{2}{*}{ Walker } & Yes & 12 & 24 & 00 & 00 & $0.026^{*}$ \\
\hline & No & 38 & 76 & 10 & 00 & \\
\hline Staff/ & Yes & 23 & 46 & 06 & 60 & 0.418 \\
\hline Personnel & No & 27 & 54 & 04 & 40 & \\
\hline Activity during th & me of $f$ & & & & & \\
\hline Bathing & Yes & 02 & 04 & 01 & 10 & 0.468 \\
\hline & No & 48 & 96 & 09 & 90 & \\
\hline
\end{tabular}




\begin{tabular}{|c|c|c|c|c|c|c|}
\hline \multirow{2}{*}{$\begin{array}{l}\text { Housework } \\
\text { (obstacles) }\end{array}$} & Yes & 19 & 38 & 04 & 40 & \multirow[t]{2}{*}{0.906} \\
\hline & No & 31 & 62 & 06 & 60 & \\
\hline \multirow[t]{2}{*}{ Climbing stairs } & Yes & 01 & 02 & 01 & 10 & \multirow[t]{2}{*}{0.267} \\
\hline & No & 49 & 98 & 09 & 90 & \\
\hline \multirow[t]{2}{*}{ Transferring } & Yes & 00 & 00 & 03 & 30 & \multirow[t]{2}{*}{$0.001^{*}$} \\
\hline & No & 50 & 100 & 07 & 70 & \\
\hline \multirow[t]{2}{*}{ Ambulation } & Yes & 34 & 68 & 06 & 60 & \multirow[t]{2}{*}{0.628} \\
\hline & No & 16 & 32 & 04 & 40 & \\
\hline \multicolumn{7}{|l|}{ Location of fall } \\
\hline \multirow[t]{2}{*}{ Bathroom } & Yes & 05 & 10 & 00 & 00 & \multirow[t]{2}{*}{0.167} \\
\hline & No & 45 & 90 & 10 & 100 & \\
\hline \multirow{2}{*}{ Outside house } & Yes & 21 & 42 & 04 & 40 & \multirow[t]{2}{*}{0.907} \\
\hline & No & 29 & 58 & 06 & 60 & \\
\hline \multirow{2}{*}{$\begin{array}{l}\text { Other rooms inside } \\
\text { the house }\end{array}$} & Yes & 25 & 50 & 06 & 60 & \multirow[t]{2}{*}{0.562} \\
\hline & No & 25 & 50 & 04 & 40 & \\
\hline \multirow{2}{*}{$\begin{array}{l}\text { Street/ } \\
\text { Road }\end{array}$} & Yes & 10 & 20 & 02 & 20 & \multirow[t]{2}{*}{1.000} \\
\hline & No & 40 & 80 & 08 & 80 & \\
\hline \multirow{2}{*}{$\begin{array}{l}\text { Threshold at the door } \\
\text { entrance }\end{array}$} & Yes & 09 & 18 & 02 & 20 & 0.882 \\
\hline & No & 41 & 82 & 08 & 80 & \\
\hline Vehicle related & Yes & 09 & 18 & 00 & 00 & 0.058 \\
\hline & No & 41 & 82 & 10 & 100 & \\
\hline Causes of falls & & & & & & \\
\hline Hypertension & Yes & 09 & 18 & 06 & 60 & $0.005^{*}$ \\
\hline & No & 41 & 82 & 04 & 40 & \\
\hline Dizziness & Yes & 17 & 34 & 05 & 50 & 0.345 \\
\hline & No & 33 & 66 & 05 & 50 & \\
\hline Imbalance & Yes & 34 & 68 & 10 & 100 & $0.009 *$ \\
\hline & No & 16 & 32 & 00 & 00 & \\
\hline Slips & Yes & 30 & 60 & 04 & 40 & 0.246 \\
\hline & No & 20 & 40 & 06 & 60 & \\
\hline Trips & Yes & 02 & 04 & 00 & 00 & 0.389 \\
\hline & No & 48 & 96 & 10 & 100 & \\
\hline Vehicular accidents & Yes & 06 & 12 & 00 & 00 & 0.128 \\
\hline & No & 44 & 88 & 10 & 100 & \\
\hline Injuries sustained dy & to fall & & & & & \\
\hline Abrasions & Yes & 07 & 14 & 00 & 00 & 0.098 \\
\hline & No & 43 & 86 & 10 & 100 & \\
\hline Contusions & Yes & 05 & 10 & 00 & 00 & 0.167 \\
\hline & No & 45 & 90 & 10 & 100 & \\
\hline Fractures & Yes & 27 & 54 & 05 & 50 & 0.817 \\
\hline & No & 23 & 46 & 05 & 50 & \\
\hline Dislocation of joints & Yes & 09 & 18 & 01 & 10 & 0.514 \\
\hline & No & 41 & 82 & 09 & 90 & \\
\hline Sprains & Yes & 23 & 46 & 01 & 10 & $0.022 *$ \\
\hline & No & 27 & 54 & 09 & 90 & \\
\hline Treatment received & & & & & & \\
\hline $1^{\text {st }}$ aid & Yes & 45 & 90 & 10 & 100 & 0.167 \\
\hline & No & 05 & 10 & 00 & 00 & \\
\hline Hospital admission & Yes & 43 & 86 & 10 & 100 & 0.098 \\
\hline & No & 07 & 14 & 00 & 00 & \\
\hline OPD consultation & Yes & 46 & 92 & 10 & 100 & 0.218 \\
\hline & No & 04 & 08 & 00 & 00 & \\
\hline Fears of falls & & & & & & \\
\hline Restriction of daily & Yes & 27 & 54 & 06 & 60 & 0.727 \\
\hline activities & No & 23 & 46 & 04 & 40 & \\
\hline
\end{tabular}


Table 3: Gender with determinants of falls

\begin{tabular}{|c|c|c|c|c|c|c|}
\hline \multirow[t]{2}{*}{ Determinants } & & \multicolumn{2}{|c|}{ Male } & \multicolumn{2}{|c|}{ Female } & \multirow[t]{2}{*}{ P value } \\
\hline & & $\mathbf{N}$ & $(\%)$ & $\mathbf{N}$ & $(\%)$ & \\
\hline \multicolumn{7}{|c|}{ Health Status at the time of fall } \\
\hline \multirow{2}{*}{$\begin{array}{l}\text { Hypertension } \\
\text { (giddiness) }\end{array}$} & Yes & 07 & 31.8 & 12 & 34.2 & \multirow[t]{2}{*}{0.850} \\
\hline & No & 15 & 68.2 & 25 & 65.8 & \\
\hline \multirow[t]{2}{*}{ Parkinson's disease } & Yes & 00 & 00 & 01 & 2.6 & \multirow[t]{2}{*}{0.337} \\
\hline & No & 22 & 100 & 37 & 97.4 & \\
\hline \multirow{2}{*}{$\begin{array}{l}\text { Joint pain } \\
\text { (STIFFNESS) }\end{array}$} & Yes & 13 & 59.1 & 16 & 42.1 & \multirow[t]{2}{*}{0.205} \\
\hline & No & 09 & 40.9 & 22 & 57.9 & \\
\hline \multirow[t]{2}{*}{ Memory loss } & Yes & 04 & 18.2 & 02 & 6.3 & \multirow[t]{2}{*}{0.116} \\
\hline & No & 18 & 81.8 & 36 & 94.7 & \\
\hline \multirow{2}{*}{$\begin{array}{l}\text { Diabetes Mellitus } \\
\text { (peripheral neuropathy) }\end{array}$} & Yes & 03 & 13.6 & 03 & 7.9 & \multirow[t]{2}{*}{0.482} \\
\hline & No & 19 & 86.4 & 35 & 92.1 & \\
\hline \multirow{2}{*}{$\begin{array}{l}\text { Vertigo/ } \\
\text { Giddiness }\end{array}$} & Yes & 14 & 63.6 & 31 & 81.6 & \multirow[t]{2}{*}{0.122} \\
\hline & No & 08 & 36.4 & 07 & 18.4 & \\
\hline \multirow[t]{2}{*}{$\mathrm{H} / \mathrm{O}$ fractures } & Yes & 08 & 36.4 & 08 & 21.1 & \multirow[t]{2}{*}{0.196} \\
\hline & No & 14 & 63.6 & 30 & 78.9 & \\
\hline Depression & Yes & 01 & 4.5 & 00 & 00 & 0.154 \\
\hline & No & 21 & 95.5 & 38 & 100 & \\
\hline Seizures & Yes & 00 & 00 & 01 & 1.7 & 0.337 \\
\hline & No & 22 & 100 & 37 & 97.4 & \\
\hline Antihistamines & Yes & 01 & 4.5 & 02 & 5.3 & 0.902 \\
\hline & No & 21 & 95.5 & 36 & 94.7 & \\
\hline Mobility aids during falls & & & & & & \\
\hline Cane/ & Yes & 12 & 54.5 & 14 & 36.8 & 0.182 \\
\hline Walking stick & No & 10 & 45.5 & 24 & 63.2 & \\
\hline Walker & Yes & 04 & 18.2 & 08 & 21.1 & 0.788 \\
\hline & No & 18 & 81.8 & 30 & 78.9 & \\
\hline Staff/ & Yes & 14 & 63.6 & 15 & 39.5 & 0.071 \\
\hline Personnel & No & 08 & 36.4 & 23 & 60.5 & \\
\hline Activity during the time 0 & & & & & & \\
\hline Bathing & Yes & 01 & 4.5 & 02 & 6.3 & 0.902 \\
\hline & No & 21 & 95.5 & 36 & 94.7 & \\
\hline Housework & Yes & 06 & 27.3 & 17 & 44.7 & 0.180 \\
\hline (obstacles) & No & 16 & 72.7 & 21 & 55.3 & \\
\hline Climbing stairs & Yes & 01 & 4.5 & 01 & 2.6 & 0.696 \\
\hline & No & 21 & 95.5 & 37 & 97.4 & \\
\hline Transferring & Yes & 01 & 4.5 & 02 & 5.3 & 0.902 \\
\hline & No & 21 & 95.5 & 36 & 94.7 & \\
\hline Ambulation & Yes & 17 & 77.3 & 23 & 60.5 & 0.185 \\
\hline & No & 05 & 22.7 & 15 & 39.5 & \\
\hline Bathroom & Yes & 01 & 4.5 & 04 & 10.5 & 0.399 \\
\hline & No & 21 & 95.5 & 34 & 89.5 & \\
\hline Outside house & Yes & 11 & 50 & 14 & 36.8 & 0.319 \\
\hline & No & 11 & 50 & 24 & 63.2 & \\
\hline Other rooms inside the & Yes & 09 & 40.9 & 22 & 57.9 & 0.205 \\
\hline house & No & 13 & 59.1 & 16 & 42.1 & \\
\hline Street/ & Yes & 06 & 27.3 & 06 & 15.8 & 0.290 \\
\hline Road & No & 16 & 72.7 & 32 & 84.2 & \\
\hline Threshold at the door & Yes & 04 & 18.2 & 07 & 18.4 & 0.982 \\
\hline entrance & No & 18 & 81.8 & 31 & 81.6 & \\
\hline Vehicle related & Yes & 06 & 27.3 & 03 & 7.9 & $0.047^{*}$ \\
\hline & No & 16 & 72.7 & 35 & 92.1 & \\
\hline
\end{tabular}




\begin{tabular}{|c|c|c|c|c|c|c|}
\hline \multirow[t]{2}{*}{ Hypertension } & Yes & 05 & 22.7 & 10 & 26.3 & \multirow[t]{2}{*}{0.757} \\
\hline & No & 17 & 77.3 & 28 & 73.7 & \\
\hline \multirow[t]{2}{*}{ Dizziness } & Yes & 08 & 36.4 & 14 & 36.8 & \multirow[t]{2}{*}{0.970} \\
\hline & No & 14 & 63.6 & 24 & 63.2 & \\
\hline \multirow[t]{2}{*}{ Imbalance } & Yes & 15 & 68.2 & 29 & 76.3 & \multirow[t]{2}{*}{0.492} \\
\hline & No & 07 & 31.8 & 09 & 23.7 & \\
\hline \multirow[t]{2}{*}{ Slips } & Yes & 13 & 59.1 & 21 & 55.3 & \multirow[t]{2}{*}{0.773} \\
\hline & No & 09 & 40.9 & 17 & 44.7 & \\
\hline \multirow[t]{2}{*}{ Trips } & Yes & 01 & 4.5 & 01 & 2.6 & \multirow[t]{2}{*}{0.696} \\
\hline & No & 21 & 95.5 & 37 & 97.4 & \\
\hline \multirow{2}{*}{ Vehicular accidents } & Yes & 04 & 18.2 & 02 & 6.3 & \multirow[t]{2}{*}{0.116} \\
\hline & No & 18 & 81.8 & 36 & 94.7 & \\
\hline \multicolumn{7}{|c|}{ Injuries sustained due to fall } \\
\hline \multirow[t]{2}{*}{ Abrasions } & Yes & 05 & 22.7 & 02 & 5.3 & \multirow[t]{2}{*}{$0.046^{*}$} \\
\hline & No & 17 & 77.3 & 36 & 94.7 & \\
\hline \multirow[t]{2}{*}{ Contusions } & Yes & 04 & 18.2 & 01 & 2.6 & \multirow[t]{2}{*}{$0.038^{*}$} \\
\hline & No & 18 & 81.8 & 37 & 97.4 & \\
\hline \multirow[t]{2}{*}{ Fractures } & Yes & 09 & 40.9 & 23 & 60.5 & \multirow[t]{2}{*}{0.142} \\
\hline & No & 13 & 59.1 & 15 & 39.5 & \\
\hline \multirow[t]{2}{*}{ Dislocation of joints } & Yes & 02 & 9.1 & 08 & 21.1 & \multirow[t]{2}{*}{0.213} \\
\hline & No & 20 & 90.9 & 30 & 78.9 & \\
\hline \multirow[t]{2}{*}{ Sprains } & Yes & 09 & 40.9 & 15 & 39.5 & \multirow[t]{2}{*}{0.913} \\
\hline & No & 13 & 59.1 & 23 & 60.5 & \\
\hline \multicolumn{7}{|l|}{ Treatment received } \\
\hline \multirow[t]{2}{*}{$1^{\text {st }}$ aid } & Yes & 18 & 81.8 & 37 & 97.4 & \multirow[t]{2}{*}{$0.038 *$} \\
\hline & No & 04 & 18.2 & 01 & 2.6 & \\
\hline \multirow[t]{2}{*}{ Hospital admission } & Yes & 19 & 86.4 & 34 & 89.5 & \multirow[t]{2}{*}{0.720} \\
\hline & No & 03 & 13.6 & 04 & 10.5 & \\
\hline \multirow[t]{2}{*}{ OPD consultation } & Yes & 21 & 95.5 & 35 & 92.1 & 0.607 \\
\hline & No & 01 & 4.5 & 03 & 7.9 & \\
\hline Fears of falls & & & & & & \\
\hline Restriction of daily & Yes & 07 & 31.8 & 26 & 68.4 & $0.006^{*}$ \\
\hline activities & No & 15 & 58.2 & 12 & 31.6 & \\
\hline
\end{tabular}

Table 4: Analysis of association between repeated history of fall and various determinants

\begin{tabular}{|c|c|c|c|c|c|c|c|}
\hline \multicolumn{2}{|c|}{ Determinants } & \multicolumn{3}{|c|}{ Repeated history of fall } & \multirow{2}{*}{\multicolumn{2}{|c|}{ p value }} & \multirow[t]{3}{*}{ Comments } \\
\hline & & \multicolumn{2}{|c|}{ Yes } & \multirow{2}{*}{\begin{tabular}{|l|} 
No \\
$\mathrm{N}(28)$
\end{tabular}} & & & \\
\hline & & $\mathrm{N}(32)$ & $\%$ & & $\%$ & & \\
\hline \multirow[t]{2}{*}{ Vertigo } & Yes & 21 & 70.0 & 09 & 30.0 & \multirow{2}{*}{$\begin{array}{c}0.010^{*} \\
0.019\end{array}$} & \multirow[t]{2}{*}{$\chi^{2}=6.696$} \\
\hline & No & 11 & 36.7 & 19 & 63.3 & & \\
\hline \multirow[t]{2}{*}{ Sedatives } & Yes & 01 & 33.3 & 02 & 66.6 & \multirow{2}{*}{$\begin{array}{l}0.276 \\
0.594\end{array}$} & \multirow[t]{2}{*}{$\chi^{2}=0.508$} \\
\hline & No & 31 & 54.4 & 26 & 45.6 & & \\
\hline \multirow[t]{2}{*}{ HTN } & Yes & 11 & 73.3 & 04 & 26.7 & \multirow{2}{*}{$\begin{array}{l}0.073 \\
0.084\end{array}$} & \multirow[t]{2}{*}{$\chi^{2}=3.214$} \\
\hline & No & 21 & 46.7 & 24 & 53.3 & & \\
\hline \multirow[t]{2}{*}{ Joint pain } & Yes & 15 & 51.7 & 14 & 48.3 & \multirow{2}{*}{$\begin{array}{l}0.809 \\
1.000\end{array}$} & \multirow[t]{2}{*}{$\chi^{2}=0.058$} \\
\hline & No & 17 & 54.8 & 14 & 45.2 & & \\
\hline \multirow[t]{2}{*}{ Memory loss } & Yes & 03 & 50.0 & 03 & 50.0 & \multirow{2}{*}{$\begin{array}{l}0.863 \\
1.000\end{array}$} & \multirow[t]{2}{*}{$\chi^{2}=0.030$} \\
\hline & No & 29 & 53.7 & 25 & 46.3 & & \\
\hline \multirow[t]{2}{*}{$\mathrm{DM}$} & Yes & 03 & 50.0 & 03 & 50.0 & \multirow{2}{*}{$\begin{array}{l}0.863 \\
1.000\end{array}$} & \multirow[t]{2}{*}{$\chi^{2}=0.030$} \\
\hline & No & 29 & 53.7 & 25 & 46.3 & & \\
\hline \multirow[t]{2}{*}{ Vertigo/giddiness } & Yes & 29 & 64.4 & 16 & 35.6 & \multirow{2}{*}{$\begin{array}{c}0.003 * \\
0.006\end{array}$} & \multirow[t]{2}{*}{$\chi^{2}=8.929$} \\
\hline & No & 03 & 20.0 & 12 & 80.0 & & \\
\hline \multirow[t]{2}{*}{$\mathrm{H} / \mathrm{O}$ fractures } & Yes & 04 & 25.0 & 12 & 75.0 & \multirow{2}{*}{$\begin{array}{c}0.008^{*} \\
0.010 \\
\end{array}$} & \multirow[t]{2}{*}{$\chi^{2}=7.037$} \\
\hline & No & 28 & 63.6 & 16 & 36.4 & & \\
\hline Antihistaminic & Yes & 02 & 66.7 & 01 & 33.3 & 0.635 & $\chi^{2}=0.226$ \\
\hline
\end{tabular}




\begin{tabular}{|l|c|c|c|c|c|c|c|}
\hline & No & 30 & 52.6 & 27 & 47.4 & 1.000 & \\
\hline \multirow{2}{*}{ Dizziness } & Yes & 16 & 72.7 & 06 & 27.3 & $0.022^{*}$ & $\chi^{2}=5.249$ \\
\cline { 2 - 7 } & No & 16 & 42.1 & 22 & 57.9 & 0.032 & \\
\hline Imbalance & Yes & 28 & 63.6 & 16 & 36.4 & $0.008^{*}$ & $\chi^{2}=7.037$ \\
\cline { 2 - 6 } & No & 04 & 25.0 & 12 & 75.0 & 0.010 & \\
\hline
\end{tabular}

\section{Discussion}

In the study, a total of 60 geriatric participants were there. 50 participants $(83.33 \%)$ were in the age group 60-74 years, and $10(16.67 \%)$ were above 75 years age. Among them, $22(36.67 \%)$ were males and $38(63.33 \%)$ were females. Among 50 young old (60-75 years age), males were $18(36 \%)$ and females were 32 (64\%), whereas among 10 old elderly (>76 years age) males were $04(40 \%)$ and females were $06(60 \%)$; which is similar to a study done by Kolli SS et $\mathrm{a}^{10}$ in Vijaypur in 2015 showed that among 50 participants with history of fall, $28(56 \%)$ were females and 22 (44\%) were males. Results from study done by Patil SS et $\mathrm{al}^{7}$ in 2011 in Bangalore, Karnataka revealed that among 416 participated elders, $88.4 \%$ were in $60-75$ years age similar to the current study. And 9.2\%, 2.4\% respectively between age groups of 76-85 years and $>85$ years. $64.4 \%$ females as compared to $35.6 \%$ males similar to the current study.

Among the study participants, 32 (53.3\%) had repeated falls from the current study, where as a study done in Assam (2013) by Agarwalla R et al, ${ }^{6}$ showed $41.9 \%$ had at least 1 episode of fall and $14 \%$ had repeated fall. $29.8 \%$ elderly had previous history of fall as per the study done by Patil SS et $\mathrm{al}^{7}$ in Bangalore, 2011. There was no significant difference in fall rate of $31.3 \%$ females and $27.02 \%$ males ( $p>0.05$ ) which is in contrast to the current study showing fall rate of $15 \%$ among males and 23\% females and is not significant $(p>0.05)$. Whereas, the study done by Kolli SS et $\mathrm{al}^{10}$ in Vijaypur in 2015 revealed 12\% patients had previous history of fall.

According to the study done by Patil SS et $\mathrm{al}^{7}$ in Bangalore, 2011 showed that outdoor falls were 57.2\% as compared to indoor falls $42.8 \%$. Among the outdoor falls $63.3 \%$ falls were observed on footpath. Among indoor falls, $30.1 \%$ falls were in the bathroom, followed by $22.6 \%$ falls while using stairs. Current study showed that among falls outdoor and indoor came to be 50\% each. And among outdoor falls, 92\% were outside house on the footpath. Among indoor falls, $28.57 \%$ had fall in bathroom.

In the current study, majority of falls had occurred in the evening $(41.67 \%$ ), followed by $26.67 \%$ in the morning, $18.33 \%$ in the afternoon and $13.33 \%$ in the night. In contrast to this a study done by Patil SS et $\mathrm{al}^{7}$ in Bangalore, 2011 revealed majority i.e., 67 (54\%) of the elderly had falls in the morning, followed by 41 $(33.2 \%)$ in the evening, $15(12 \%)$ in the afternoon and only $1(0.8 \%)$ in the night.

In the current study, Hypertension as a cause for fall found significant from the current study among old elderly (60\%). There are $33.3 \%$ geriatric study participants having hypertension and were on treatment, when compared to a study conducted by Chinnakali $\mathrm{P}$ et al, ${ }^{11}$ Puducherry India 2016, has prevalence of hypertension as $40.5 \%$. In the current study, hypertension among the history of repeated falls were $52.3 \%$ and is not statistically significant. Similarly, a study conducted by Kolli SS et $\mathrm{al}^{10}$ in Vijaypur in 2015 showed that all 6 of the participants having history of repeated falls were having hypertension.

Vehicle related or fall from vehicle while transferring has found significant among elderly males $(27.3 \%)$. First aid is the main treatment received after fall is found from the current study (97.4\%). Restriction of the daily activity seen more among female geriatric study population from the current study $(68.4 \%)$ in the current study.

In the current study, there are $64 \%$ elderly female and $60 \%$ of very elderly female. Among the illiterates in the study population, there are $71.1 \%$ females, which coincides with the statistics given in Elderly India 2016 $(71.5 \%) .{ }^{12}$ Similarly, among marital status of married, there is $50 \%$ distribution each for both males and females, again coincide the data with the statistics given in Elderly India 2016.

\section{Conclusion}

The present study shows the falls, causes for falls, and its consequences in the form of one or the other injuries like abrasions, contusions, fractures, dislocations and sprains increasing the morbidity status of the elderly. It also leads to decreased activity, loss of independence, leading to psychological distress, fear and restriction of daily activities. There is a need for rehabilitation of the elderly. Regular health checkups camps needed. Also focus to be on the determinants of falls, fractures and frailty. Emphasis has to be given on the environmental aspects like housing, with clutters, inadequate lighting, threshold at the entrance of the house, avoiding the slippery floor in the house, keeping the rails on the side walls while climbing stairs, also rail in the toilets to be encouraged to avoid falls in the elderly. Elderly become frail as the days passes off.

Recommendations: It is from the current study; proper rehabilitation is necessary. Awareness of the health check-up camps needed. Proper use of mobility aids to avoid falls and education regarding the same.

Acknowledgements: to the study participants

Conflicts of Interest: None 


\section{Funding for the Study: None}

\section{References}

1. World Health Organization. WHO global report on falls prevention in older age. 2007. World Health Organization. 2015:1-7.

2. Suryakantha AH. Community medicine with recent advances. $4^{\text {th }}$ ed. New Delhi: Jaypee the Health Sciences Publisher; 2017. p. 775.

3. Fillit H, Butler RN: The frailty identity crisis. J Am Geriatr Soc 2009,57(2):348-352

4. Try this: Best Practices in Nursing Care to Older Adults, The Hartford Institute for Geriatric Nursing, New York University, College of Nursing, www.hartfordign.org.

5. Fried LP, Tangen CM, Walston J, et al. Frailty in older adults: evidence for a phenotype. J Gerontol A BiolSci Med Sci 2001;56:M146-M156.

6. Agarwalla R, Saikia AM, Pathak R, Islam F, Borah M, Parashar M. A Cross Sectional Study on Assessment of Falls in Community Dwelling Elderly of Assam. Ntl J Community Med 2016;7(5):368-371.

7. Patil SS, Suryanarayana SP. Circumstances and Consequences of Falls in Community-Living Elderly in North Bangalore Karnataka. Journal of Krishna Institute of Medical Sciences (JKIMSU). 2015 Oct 1;4(4).
8. Tinetti ME, Speechley M, Ginter SF. Risk factors for falls among elderly persons living in the community. New England journal of medicine. 1988 Dec 29;319(26):1701-7.

9. Sirohi A, Kaur R, Goswami AK, Mani K, Nongkynrih B, Gupta SK. A study of falls among elderly persons in a rural area of Haryana. Indian J Public Health 2017;61:99-104.

10. Kolli SS et al. Clinical profile of the elderly. Scholars Journal of Applied Medical Sciences (SJAMS) 2017;5(2C):513-516.

11. Chinnakali P, Mohan B, Upadhyay RP, Singh AK, Srivastava R, Yadav K. Hypertension in the elderly: prevalence and health seeking behavior. North American journal of medical sciences. 2012 Nov;4(11):558.

12. National Sample Survey Office. Elderly in India. Minist Statisitcs Program Implementation, Government of India [Internet]. 2016;1-95. Available from: http://mospi.nic.in/sites/default/files/publication_reports/Dis abled_persons_in_India_206.pdf 